\title{
Treating Second Breast Events After Breast-Conserving Surgery for Ductal Carcinoma in Situ
}

\author{
Michael J. Hassett, MD, MPH ${ }^{\mathrm{a}, \mathrm{b}}$; Wei Jiang, MSc; Melissa E. Hughes, MSc ${ }^{\mathrm{a}}$; Stephen Edge, MD \\ Sara H. Javid, MD'; Joyce C. Niland, $\mathrm{PhD}^{f}$; Richard Theriault, MD; ; Yu-Ning Wong, MD; \\ Deborah Schrag, MD ${ }^{\mathrm{a}, \mathrm{b}}$; and Rinaa S. Punglia, MD, $\mathrm{MPH}^{\mathrm{b}, \mathrm{i}}$
}

\begin{abstract}
Background: Because of screening mammography, the number of ductal carcinoma in situ (DCIS) survivors has increased dramatically. DCIS survivors may face excess risk of second breast events (SBEs). However, little is known about SBE treatment or its relationship to initial DCIS care. Methods: Among a prospective cohort of women who underwent breast-conserving surgery (BCS) for DCIS from 1997 to 2008 at institutions participating in the NCCN Outcomes Database, we identified SBEs, described patterns of care for SBEs, and examined the association between DCIS treatment choice and SBE care. Using multivariable regression, we identified features associated with use of mastectomy, radiation therapy (RT), or antiestrogen therapy (AET) for SBEs. Results: Of 2,939 women who underwent BCS for DCIS, $83 \%$ received RT and $40 \%$ received AET. During the median follow-up of 4.2 years, 200 women (6.8\%) developed an SBE (55\% ipsilateral, $45 \%$ invasive). SBEs occurred in $6 \%$ of women who underwent RT for their initial DCIS versus $11 \%$ who did not. Local treatment for these events included BCS $(10 \%), \mathrm{BCS} / \mathrm{RT}(30 \%)$, mastectomy $(53 \%)$, or none $(6 \%)$; only $28 \%$ of patients received AET. Independent predictors of RT or mastectomy for SBEs included younger age, shorter time to SBE diagnosis, and RT or AET for the initial DCIS. Conclusions: A sizable proportion of patients with SBEs were treated with mastectomy, most especially those who previously received RT for their initial DCIS and those who developed an ipsilateral SBE. Despite the occurrence of an SBE, relatively few patients received AET. Future studies should investigate optimal treatment approaches for SBEs, including the benefit of mastectomy versus lumpectomy for an ipsilateral SBE and the benefit of AET for a hormone-receptor-positive SBE contingent on AET use for the initial DCIS diagnosis.
\end{abstract}

J Natl Compr Canc Netw 2018;16(4):387-394 doi: $10.6004 / j n c c n .2018 .7003$

\begin{abstract}
As the incidence of ductal carcinoma in situ (DCIS) has increased, so has the number of DCIS survivors. ${ }^{1}$ Most patients with DCIS are treated with breast-conserving surgery (BCS). ${ }^{2}$ Radiation therapy (RT) and antiestrogen therapy (AET) have been shown to reduce the risk of recurrence, ${ }^{3,4}$ and are recommended by the NCCN Guidelines. ${ }^{5}$ However, relative survival remains
\end{abstract}

\footnotetext{
aDivision of Population Sciences, Dana-Farber Cancer Institute, Boston, Massachusetts; 'barvard Medical School, Boston, Massachusetts; 'Department of Surgery, Brigham \& Women's Hospital, Boston, Massachusetts; dDepartment of Surgical Oncology, Roswell Park Comprehensive Cancer Center, Buffalo, New York; eUniversity of Washington, Seattle, Washington; ${ }^{f}$ City of Hope Comprehensive Cancer Center, Duarte, California; ${ }^{9} T h e$ University of Texas MD Anderson Cancer Center, Houston, Texas; ' $F$ Fox Chase Cancer Center, Philadelphia, Pennsylvania; and 'Department of Radiation Oncology, Dana-Farber Cancer Institute, Boston, Massachusetts.

Submitted July 1, 2017; accepted for publication January 3, 2018.

The authors have disclosed that they have no financial interests, arrangements, affiliations, or commercial interests with the manufacturers of any products discussed in this article or their competitors.
}

extremely high $(>98 \%)$ with or without these additional treatments. ${ }^{6-11}$ Some providers and patients believe the benefits of RT and AET are not meaningful enough to warrant their potential adverse effects. Not surprisingly, patterns of use for these treatment options vary, suggesting that there still is not universal consensus regarding optimal treatment of DCIS. ${ }^{12,13}$

This study was supported by contract \# HHSA290200500161 from AHRQ. Dr. Hassett received salary support from a Susan G. Komen for the Cure Career Catalyst Award. The views expressed in this article are those of the authors, and no official endorsement by AHRQ or the U.S. Department of Health and Human Services is intended or should be inferred.

Author contributions: Study concept and design: Hassett, Punglia.

Data acquisition: Hassett, Edge, Javid, Niland, Wong. Data analysis and interpretation: Hassett, Jiang, Hughes, Punglia. Manuscript preparation and review: Hassett, Jiang, Hughes, Edge, Javid, Niland, Theriault, Wong, Schrag, Punglia. Administrative and financial support: Hassett, Schrag, Punglia.

Correspondence: Michael J. Hassett, MD, MPH, Division of Population Sciences, Dana-Farber Cancer Institute, 450 Brookline Avenue, Boston, MA 02215-5450. Email: michael_hassett@dfci.harvard.edu 
DCIS survivors are at increased risk for developing second breast events (SBEs). ${ }^{9}{ }^{14-16}$ Recently, we compared the pathologic characteristics of initial DCIS and SBEs in 2 separate data sets, and found similarities with respect to grade and hormone receptor (HR) status. ${ }^{17,18}$ Studies suggest that approximately half of all SBEs are invasive. ${ }^{14-16}$ Compared with patients first diagnosed with breast cancer, those with SBEs tend to be older and have more comorbid conditions, and may have a greater risk of distant recurrence. ${ }^{17}$ Unfortunately, relatively little is known regarding the optimal approach to treating SBEs. Should we extrapolate from studies of patients with newly diagnosed DCIS/cancer, or do the unique aspects of SBEs necessitate different treatment approaches? How do treatments provided for an initial DCIS, which can be quite variable, impact treatments offered to patients who develop an SBE?

Using a large cohort of patients who underwent BCS for DCIS, we sought to describe the treatments provided to patients who experienced an SBE and to identify independent determinants of mastectomy or AET for SBEs. We hypothesized that younger patients and those who received RT or AET for their initial DCIS treatment could be more likely to receive mastectomy or AET for their SBE therapy. Moreover, patients who received no RT for their initial DCIS could be less like to receive potentially beneficial treatments for their SBE.

\section{Methods}

\section{Data Source}

This cohort study relied on data from the NCCN Outcomes Database for Breast Cancer. ${ }^{19}$ From 1997 to 2012, participating institutions prospectively collected patient and tumor characteristics, treatments, and outcomes for women with newly diagnosed breast cancer. Data collection included a baseline patient survey and a series of prospectively scheduled medical record reviews. Abstraction involved rigorous, regularly scheduled data quality assurance checks. Patients were considered lost to follow-up if they had no visit to the treating site for $>2$ consecutive years.

A total of 9 NCCN Member Institutions contributed data to this analysis: City of Hope Comprehensive Cancer Center, Dana-Farber Cancer Institute, Fox Chase Cancer Center, Moffitt Cancer Center,
Massachusetts General Hospital Cancer Center, The Ohio State University Comprehensive Cancer Center - James Cancer Hospital and Solove Research Institute, Roswell Park Comprehensive Cancer Center, The University of Texas MD Anderson Cancer Center, and University of Michigan Comprehensive Cancer Center. The Institutional Review Board at each center approved the parent study, data collection procedures, transmission methods, and storage protocols. This analysis was granted an exemption by the Dana-Farber/Harvard Cancer Center Office for Human Research Studies.

\section{Cohort Derivation and Variable Definitions}

All patients were diagnosed with DCIS and treated with BCS from July 1, 1997 to December 31, 2008, to allow sufficient follow-up time to identify SBE events. Information collected at the initial DCIS diagnosis included sociodemographic characteristics, menopausal status, cancer stage, tumor pathology, and local treatments. Information gathered from subsequent chart reviews included systemic treatments and recurrence. Comorbidities at presentation were scored using the Charlson comorbidity index ${ }^{20}$ or its modification developed by Katz et al. ${ }^{21}$

Surgical margins were considered negative if there was no tumor within $2 \mathrm{~mm}$ of ink, or close/ positive if there was tumor within $2 \mathrm{~mm}$. Grade was categorized as 1 (well-differentiated/low); 2 (moderately differentiated/intermediate); or 3 (poorly differentiated/high). Immunohistochemistry was used to categorize the estrogen receptor (ER) and progesterone receptor $(\mathrm{PR})$ status as positive or negative. If either ER or PR was positive, the tumor was considered HR-positive. For DCIS cases, the database did not collect tumor size and margin status before July 1, 1999, or HR status before April 1, 2003.

Use of AET for initial DCIS diagnosis was defined as documentation of therapy with a selective ER modulator, aromatase inhibitor, or gonadotropin-releasing hormone agonist lasting at least 30 days after diagnosis and starting $>30$ days before any SBE. Descriptive statistics for the use of AET included the proportion of patients who received therapy, median time to initiation of therapy, and median duration of therapy. Duration of AET was calculated from the date of initiation to the date of discontinuation, SBE diagnosis, last follow-up, or death, whichever came first. 
For patients who developed a SBE, the pathologic characteristics of and treatments for the event were also abstracted. SBEs were classified as ipsilateral invasive, ipsilateral noninvasive, contralateral invasive, or contralateral noninvasive (there were no distant recurrences). Time to recurrence was dichotomized according to the median time since initial DCIS diagnosis. Use of AET for the recurrence was defined as any documentation of AET lasting at least 30 days after the SBE diagnosis.

\section{Statistical Analyses}

Comparisons between patients from the whole cohort (ie, DCIS treated with BCS) and the subset that developed an SBE were performed using the chi square test. Multivariable logistic regression was used to identify independent predictors of AET for the initial DCIS; candidate covariates included baseline patient and tumor characteristics (Table 1). Multivariable logistic regression was used to identify independent predictors of mastectomy and AET for SBEs. Candidate covariates for these models included baseline patient characteristics (Table 1) and tumor features at recurrence (Table 2). A variable was entered into the model if the $P$ value describing its bivariable association was $<0.1$, and retained if its $P$ value remained $<0.1$. Several prespecified, clinically relevant variables were included regardless of their $P$ values: age, size, HR status, margin, and AET after DCIS. Due to the potential for interaction between RT use for the initial DCIS and laterality of the SBE, these covariates were entered as a combined stratified variable, as were AET use for the initial DCIS and HR status of the SBE. Clustering by NCCN Member Institution was incorporated into each model. Model results were presented as odds ratios (ORs) with 95\% CIs. Statistical analyses were performed using SAS 9.3 (SAS Institute, Inc.).

\section{Results}

During the 12 years of this study, 2,939 patients with DCIS were treated with BCS. Approximately onethird were age $>60$ years at diagnosis, $16 \%$ were nonwhite/Hispanic, almost half were overweight/obese (body mass index $\geq 25 \mathrm{~kg} / \mathrm{m}^{2}$ ), and one-quarter had a comorbidity score $\geq 1$ (Table 1 ). Infrequently, the initial DCIS was large ( $\geq 2 \mathrm{~cm}$ in $10 \%)$ or had positive margins $(3 \%) ; 31 \%$ were high grade. HR status was positive in $49 \%$, but was missing in $42 \%$ of cas- es. Most women (83\%) received RT for their initial DCIS; only 40\% received AET. Of AET recipients, most (92\%) took tamoxifen, 5\% took an aromatase inhibitor, and 3\% took both. Median time from initial DCIS diagnosis to initiation of AET was 4.7 months (range, 0.1-105). The median duration of AET was 34.6 months (range, 1-110). Use of AET after initial DCIS diagnosis (36\%) and median time from initial DCIS diagnosis to initiation of AET (5.3 months; range, 0.5-105) among patients who developed an SBE were not substantially different from the full cohort. Factors independently associated with greater use of AET for initial DCIS included age $<50$ versus $\geq 60$ years (OR, $1.5 ; 95 \% \mathrm{CI}, 1.2-1.9)$ and receipt of RT versus no RT (OR, 1.7; 95\% CI, 1.3-2.3). Factors associated with lesser use of AET for initial DCIS included $\geq 1$ versus 0 comorbidity score (OR, 0.7; 95\% $\mathrm{CI}, 0.6-0.9)$ and HR-negative versus HR-positive DCIS (OR, 0.2; 95\% CI, 0.1-0.5) (supplemental eTable 1, available with this article at JNCCN.org).

At a median follow-up of 4.2 years (range, 1.3 11.4), 200 patients (6.8\%) developed an SBE-3.7\% in situ and $3.1 \%$ invasive. SBEs were categorized as 69 ipsilateral in situ (34\%), 42 ipsilateral invasive (21\%), 40 contralateral in situ (20\%), and 49 contralateral invasive $(24 \%)$ (Table 2$)$. They were also categorized as $71 \mathrm{HR}$-positive/unknown invasive (35.5\%), 98 HR-positive/unknown in situ (49.0\%), and $31 \mathrm{HR}$-negative invasive or in situ (15.5\%). SBEs occurred in $6 \%$ of women who received RT for their initial DCIS versus $11 \%$ who did not. Median time from initial DCIS to SBE was 33.5 months (range, 3.5-124). Factors independently associated with greater odds of developing an SBE included initial DCIS pathologic size $\geq 2 \mathrm{~cm}$ versus 0 to $1 \mathrm{~cm}$ (OR, 2.18; 95\% CI, 1.50-3.17); initial DCIS HR-negative versus HR-positive (OR, 2.81; 95\% CI, 2.11-3.74); and initial DCIS not treated with RT (OR, 2.38; 95\% CI, 1.73-3.26) (supplemental eTable 2). Postmenopausal status was associated with lesser odds of developing an SBE versus premenopausal status (OR, 0.43; 95\% CI, 0.23-0.79). Age, race/ethnicity, body mass index, comorbidity score, initial DCIS margin, initial DCIS grade, and initial DCIS AET were not significantly associated with greater odds of developing an SBE.

The most common local treatment for SBE was mastectomy (53\%), followed by BCS/RT (30\%) and BCS alone $(10 \%) ; 6 \%$ received no local therapy. 
Hassett et al

\begin{tabular}{|c|c|c|c|}
\hline Characteristics of Patients \& Initial DCIS Diagnoses & $\begin{array}{l}\text { All Patients } \\
\text { n (\%) }\end{array}$ & $\begin{array}{c}\text { Patients With SBEs } \\
\text { n (\%) }\end{array}$ & $P$ Value $^{\text {a }}$ \\
\hline Patients, N & 2,939 & 200 & \\
\hline Median follow-up (range), y & $4.2(1.3-11.4)$ & $6.3(1.3-11.4)$ & $<.01$ \\
\hline Age at diagnosis, y & & & .02 \\
\hline$<50$ & $901(31)$ & $78(39)$ & \\
\hline $50-60$ & $971(33)$ & $53(26)$ & \\
\hline$>60$ & $1,067(36)$ & $69(34)$ & \\
\hline Median (range) & $56(24-94)$ & $53(36-84)$ & \\
\hline Race/Ethnicity & & & .67 \\
\hline White non-Hispanic & $2,442(83)$ & $164(82)$ & \\
\hline Nonwhite/Hispanic & $481(16)$ & $36(18)$ & \\
\hline Unknown & $16(<1)$ & $0(0)$ & \\
\hline Menopausal status & & & $<.01$ \\
\hline Premenopausal & $990(34)$ & $90(45)$ & \\
\hline Postmenopausal & $1,948(66)$ & $110(55)$ & \\
\hline Unknown & $1(<1)$ & $0(0)$ & \\
\hline Body mass index, $\mathrm{kg} / \mathrm{m}^{2}$ & & & .52 \\
\hline$<25$ & $998(34)$ & $75(38)$ & \\
\hline$\geq 25$ & $1,433(49)$ & $94(47)$ & \\
\hline Unknown & $508(17)$ & $31(15)$ & \\
\hline Comorbidity score $^{b}$ & & & .72 \\
\hline 0 & $2,235(76)$ & $150(75)$ & \\
\hline$\geq 1$ & $704(24)$ & $50(25)$ & \\
\hline Year of diagnosis & & & $<.01$ \\
\hline 1997-2000 & $564(19)$ & $53(26)$ & \\
\hline 2001-2004 & $1,073(36)$ & $84(42)$ & \\
\hline $2005-2008$ & $1,302(44)$ & $63(32)$ & \\
\hline Presentation & & & .38 \\
\hline Mammography & $2,543(86.5)$ & $169(84)$ & \\
\hline Symptomatic (eg, palpation) & $396(13.5)$ & $31(16)$ & \\
\hline Pathologic size, $\mathrm{cm}$ & & & $<.01$ \\
\hline$<1$ & $1,078(37)$ & $61(30)$ & \\
\hline 1 to $<2$ & $468(16)$ & $31(16)$ & \\
\hline$\geq 2$ & $304(10)$ & $34(17)$ & \\
\hline Unknown & $1,089(37)$ & $74(37)$ & \\
\hline Margin & & & .43 \\
\hline Positive & $102(3)$ & $9(4)$ & \\
\hline Negative & $2,823(96)$ & $191(96)$ & \\
\hline Unknown/Other & $14(<1)$ & $0(0)$ & \\
\hline Grade & & & .47 \\
\hline Low & $432(15)$ & $22(11)$ & \\
\hline Intermediate & $869(30)$ & $60(30)$ & \\
\hline High & $922(31)$ & $68(34)$ & \\
\hline Unknown/Other & $716(24)$ & $50(25)$ & \\
\hline Subtype & & & .19 \\
\hline Comedo & $965(33)$ & $75(37)$ & \\
\hline Noncomedo & $1,930(66)$ & $124(62)$ & \\
\hline Unknown/Other & $44(1)$ & $1(<1)$ & \\
\hline Hormone receptor status & & & $<.01$ \\
\hline Positive & $1,446(49)$ & $61(30)$ & \\
\hline Negative & $246(8)$ & $29(14)$ & \\
\hline Unknown/Other & $1,247(42)$ & $110(55)$ & \\
\hline Radiation therapy & & & $<.01$ \\
\hline Yes & $2,433(83)$ & $145(72)$ & \\
\hline No & $496(17)$ & $55(28)$ & \\
\hline Antiestrogen therapy & & & .18 \\
\hline Any & $1,189(40)$ & $72(36)$ & \\
\hline None & $1,750(60)$ & $128(64)$ & \\
\hline
\end{tabular}

Abbreviations: DCIS, ductal carcinoma in situ; SBEs, second breast events.

${ }^{a} P<.05$ was considered significant.

${ }^{\mathrm{b}}$ Comorbidities at presentation were scored using either the medical record-based Charlson comorbidity index ${ }^{20}$ or the patient survey-based modification developed by Katz et al. ${ }^{21}$

Among patients with an ipsilateral SBE, mastectomy was a more common treatment if the initial DCIS therapy included RT versus BCS alone $(85.5 \%$ vs $54.3 \% ; \mathrm{P}<.001$ ) (Figure 1A). In contrast, among patients with a contralateral SBE, mastectomy was uncommon regardless of whether the previous DCIS treatment was BCS/RT or BCS alone $(20.3 \%$ vs $40.0 \% ; P=.072$ ). Only $28 \%$ of patients with an SBE 
Treating Second Breast Events

\begin{tabular}{|c|c|}
\hline Characteristic & $n(\%)$ \\
\hline Patients, N & 200 \\
\hline Median time from initial DCIS to SBE (range), mo & $33.5(3.5-124)$ \\
\hline \multicolumn{2}{|l|}{ Age at SBE diagnosis, $y$} \\
\hline$<50$ & $52(26)$ \\
\hline $50-60$ & $67(34)$ \\
\hline$>60$ & $81(40)$ \\
\hline Median (range) & $56.5(40.0-8.6)$ \\
\hline \multicolumn{2}{|l|}{ Type } \\
\hline In situ & $109(54)$ \\
\hline Invasive & $91(46)$ \\
\hline \multicolumn{2}{|l|}{ Location } \\
\hline Ipsilateral & $111(56)$ \\
\hline Contralateral & $89(44)$ \\
\hline \multicolumn{2}{|l|}{ Pathologic size, $\mathrm{cm}$} \\
\hline 0 to $<1$ & $59(30)$ \\
\hline 1 to $<2$ & $52(26)$ \\
\hline$\geq 2$ & $25(12)$ \\
\hline Unknown/Other & $64(32)$ \\
\hline \multicolumn{2}{|l|}{ Grade } \\
\hline Low & $17(8)$ \\
\hline Intermediate & $60(30)$ \\
\hline High & $78(39)$ \\
\hline Unknown/Other & $45(22)$ \\
\hline \multicolumn{2}{|l|}{ Hormone receptor status } \\
\hline Positive & $116(58)$ \\
\hline Negative & $31(16)$ \\
\hline Unknown/Other & $53(26)$ \\
\hline \multicolumn{2}{|l|}{ Surgery } \\
\hline BCS & $82(41)$ \\
\hline Mastectomy & $106(53)$ \\
\hline None & $12(6)$ \\
\hline \multicolumn{2}{|l|}{ Radiation therapy } \\
\hline Yes & $67(34)$ \\
\hline No & $133(66)$ \\
\hline \multicolumn{2}{|l|}{ Antiestrogen therapy } \\
\hline Any & $55(28)$ \\
\hline None & $145(72)$ \\
\hline
\end{tabular}

Abbreviations: BCS, breast-conserving surgery; DCIS, ductal carcinoma in situ; SBEs, second breast events (DCIS or invasive cancer)

received AET. Among those who received AET after recurrence, $67 \%$ took tamoxifen, $22 \%$ took an aromatase inhibitor, and $11 \%$ took both. AET was more common among patients with an SBE with HRpositive/unknown invasive disease (39\%), followed by those with HR-positive/unknown in situ disease (24\%), and lastly those with HR-negative invasive or in situ disease (10\%) (supplemental eTable 3). AET was more common among patients with an SBE who received AET for their initial DCIS compared with those who did not $(43.1 \%$ vs $18.8 \%$; $P<.01)$, regardless of whether the SBE was HR-positive or HRnegative (Figure 1B).
As expected, different factors were associated with the use of mastectomy and AET for an SBE (Table 3). Two factors were associated with lesser odds of mastectomy: younger age (OR, .037 if age $\geq 60$ vs $<50$ years; 95\% CI, 0.13-1.04), and receiving RT for the initial DCIS among patients who developed a contralateral SBE (OR, 0.27; 95\% CI, 0.17-0.44). One factor was associated with greater odds of mastectomy: having RT for the initial DCIS among patients who had an ipsilateral SBE (OR, 17.58; 95\% CI, 4.7465.24); and one factor was associated with lesser odds of AET: longer time from initial DCIS to SBE (OR, 0.37; 95\% CI, 0.16-0.86). Two factors were associated with greater odds of AET: receiving AET for the initial DCIS and having an HR-positive SBE.

\section{Discussion}

As the number of DCIS survivors increases, the number of women who develop SBEs will also likely increase. Yet, few studies have analyzed the patterns
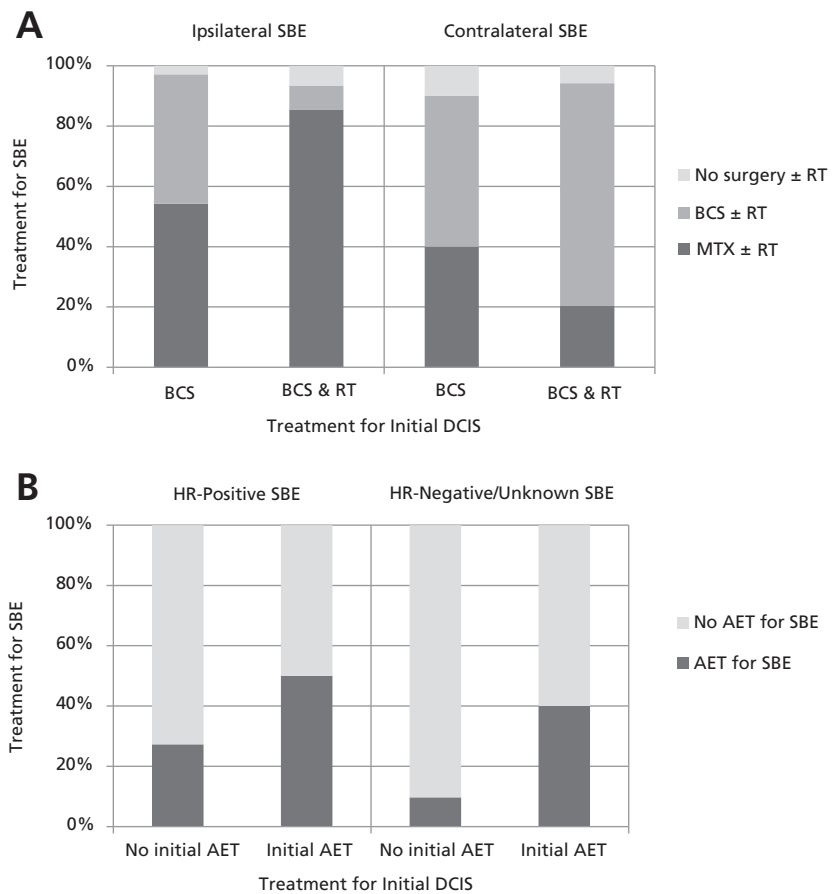

Figure 1. Treatment for SBE as a function of treatment for initial DCIS, stratified by features of the SBE. (A) Type of surgery for SBE (BCS, MTX, no surgery) as a function of the use of RT for the initial DCIS stratified, by SBE laterality. (B) Use of AET for the SBE as a function of its use for the initial DCIS, stratified by SBE HR status (positive vs negative/ unknown).

Abbreviations: AET, antiestrogen therapy; BCS, breast-conserving surgery; DCIS, ductal carcinoma in situ; HR, hormone receptor; MTX, mastectomy; RT, radiation therapy; SBE, second breast cancer. 
Hassett et al

\begin{tabular}{|c|c|c|c|c|}
\hline \multirow[b]{2}{*}{ SBE Characteristics } & \multicolumn{2}{|c|}{ Mastectomy } & \multicolumn{2}{|c|}{ AET } \\
\hline & OR $(95 \% \mathrm{Cl})$ & $P$ Value & OR $(95 \% \mathrm{Cl})$ & $P$ Value \\
\hline Age at diagnosis, y & & .02 & & .27 \\
\hline$<50$ & Ref & & Ref & \\
\hline 50 to $<60$ & $1.23(0.27-5.62)$ & & $0.82(0.37-1.85)$ & \\
\hline$\geq 60$ & $0.37(0.13-1.04)$ & & $0.39(0.10-1.58)$ & \\
\hline Year of diagnosis & & .56 & & .47 \\
\hline $1997-2000$ & Ref & & Ref & \\
\hline $2001-2004$ & $2.17(0.53-8.81)$ & & $0.49(0.07-3.34)$ & \\
\hline $2005-2008$ & $2.53(0.33-19.56)$ & & $0.37(0.07-1.99)$ & \\
\hline Pathologic size, $\mathrm{cm}$ & & .22 & & $<.01^{a}$ \\
\hline$<1$ & Ref & & Ref & \\
\hline 1 to $\leq 2$ & $4.80(0.51-45.15)$ & & $0.99(0.48-2.08)$ & \\
\hline$>2$ & $10.26(0.95-111.18)$ & & $0.33(0.05-1.99)$ & \\
\hline Unknown & $2.89(0.49-19.99)$ & & $1.80(0.55-5.92)$ & \\
\hline Time from DCIS to SBE & & .57 & & .02 \\
\hline$<$ Median & Ref & & Ref & \\
\hline$\geq$ Median & $1.41(0.43-4.70)$ & & $0.37(0.16-0.86)$ & \\
\hline Type & & .52 & & .17 \\
\hline Noninvasive & Ref & & Ref & \\
\hline Invasive & $1.64(0.36-7.39)$ & & $1.54(0.83-2.85)$ & \\
\hline AET for initial DCIS \& HR status of SBE & & .08 & & $<.01$ \\
\hline No initial AET \& HR-negative/unknown & Ref & & Ref & \\
\hline No initial AET \& HR-positive & $1.32(0.52-3.34)$ & & $5.70(1.05-31.06)$ & \\
\hline Initial AET \& HR-negative/unknown SBE & $0.44(0.12-1.66)$ & & $10.12(2.98-34.40)$ & \\
\hline Initial AET \& HR-positive SBE & $0.73(0.32-1.67)$ & & $12.67(5.69-28.22)$ & \\
\hline Radiation for initial DCIS \& location of SBE & & $<.01$ & & .66 \\
\hline No RT for initial DCIS \& ipsilateral SBE & Ref & & Ref & \\
\hline No RT for initial DCIS \& contralateral SBE & $0.90(0.24-3.39)$ & & $1.39(0.28-6.83)$ & \\
\hline RT for initial DCIS \& ipsilateral SBE & $17.58(4.74-65.24)$ & & $1.85(0.64-5.35)$ & \\
\hline RT for initial DCIS \& contralateral SBE & $0.27(0.17-0.44)$ & & $2.05(0.65-6.46)$ & \\
\hline
\end{tabular}

Abbreviations: AET, antiestrogen therapy; DCIS, ductal carcinoma in situ; HR, hormone receptor; OR, odds ratio; RT, radiation therapy; SBEs, second breast events (DCIS or invasive cancer).

aExcluding patients with unknown SBE pathologic size, the $P$ value was not significant $(P=.31)$ (OR missing vs $>2 \mathrm{~cm}: 5.48 ; 95 \% \mathrm{Cl}, 1.72-17.39$ )

of care provided to these patients. To address this knowledge gap, we described patterns of care for patients who developed an SBE after having been treated previously for DCIS with BCS. For most patients, the initial DCIS treatment also included RT $(83 \%)$. The use of RT in our cohort was similar to or slightly more common than that observed in previous community-based analyses of patients with DCIS $(60 \%-62 \%) .{ }^{22-24}$ For $40 \%$ of our cohort, initial DCIS treatment also included AET (vs $22 \%-36 \%$ in previous analyses). ${ }^{25,26}$ Having HR-positive DCIS and close/positive margins were associated with greater odds of AET use. A few trials have explored the efficacy and safety of AET without RT for breast cancer, ${ }^{6,27}$ so it was not surprising that we also observed this treatment plan among patients with DCIS.

We are not aware of other studies describing patterns of care among patients with SBEs. During a median follow-up of 4.2 years, the annualized SBE rate was $1.6 \%$. The strongest determinant of local treatment for an SBE was laterality compared with the original DCIS diagnosis. For example, patients who received RT for their initial DCIS and those who developed an ipsilateral recurrence experienced greater odds of undergoing mastectomy for their SBE. Although few clinical trials have studied local treatment options for SBEs, mastectomy is widely considered standard of care for patients who previously underwent ipsilateral BCS/RT. Therefore, the clear majority of patients $(>80 \%)$ in this cohort who developed an ipsilateral SBE received the surgical standard of care. Among patients who developed a contralateral SBE, local treatment patterns were like those that followed the initial DCIS diagnosis. For example, $82 \%$ of patients who underwent BCS for a contralateral SBE received RT (vs $83 \%$ after BCS for an initial DCIS).

AET was less common after an SBE (29\%) than after an initial DCIS diagnosis (40\%). Approximately half of the SBEs in our cohort were invasive cancers. 
Previous analyses of primary breast cancer diagnoses have shown that $20 \%$ to $40 \%$ of patients with DCIS and $50 \%$ to $70 \%$ of those with invasive cancer initiate $\mathrm{AET}{ }^{28}$ We found that younger age and higherrisk pathologic characteristics were associated with AET for the SBE, suggesting that AET was recommended to patients more likely to experience benefit. Still, only 33\% of patients with an HR-positive SBE received AET. If patients with an SBE experience an elevated risk of subsequent recurrence, as one might suspect based on having had a second occurrence of cancer, this relatively low rate of AET for SBEs could be concerning. Notably, AET for the SBE diagnosis was associated with AET for the initial DCIS diagnosis, even when the SBE was HR-negative.

Limitations of our analysis include the absence of some important pathologic characteristics (eg, grade, HR status) for a subset of patients at initial DCIS diagnosis. Some patients who received their initial DCIS care at the institution participating in this project may have received their SBE care at another institution, and therefore could have been lost to follow-up. Consequently, the subset of patients with SBEs included in this analysis may not represent the entire population of patients with SBEs. Additionally, the care provided at comprehensive cancer centers appears to be more intensive than the care provided at community-based sites. Finally, the median follow-up in our cohort was $<5$ years, precluding efforts to analyze treatment patterns for late SBE events; time from initial DCIS to SBE could impact the SBE treatment plan.

\section{Conclusions}

Much debate exists regarding how to treat an initial DCIS diagnosis, with some experts proposing that surgery and RT may not be required in selected circumstances. A decision analysis suggested that RT after BCS for DCIS increases the likelihood of eventual mastectomy, because patients who develop an ipsilateral SBE must eventually undergo a mastectomy. ${ }^{29}$ Our study lends credence to this theory, finding that the local treatment plan for an initial DCIS diagnosis influences the local treatment plan for an SBE. Whether long-term considerations should impact treatment planning after an initial DCIS diagnosis remains unclear. Ongoing studies comparing surgery versus no surgery for selected women with newly diagnosed DCIS may help determine optimal therapy for an initial DCIS. ${ }^{30}$

Unfortunately, little is known about the optimal treatment approach for patients who develop an SBE or about the risk of recurrence after treatment for an SBE. The complex range of treatments provided for an initial DCIS diagnosis and the limited data available for patients who develop an SBE challenge efforts to devise clear guidelines for women with SBEs. Among patients who develop an ipsilateral SBE, mastectomy is the recommended treatment for those who previously underwent ipsilateral RT, but few studies have explored the benefits/risks of local treatment options for ipsilateral SBEs. Perhaps lumpectomy alone would be sufficient for patients with an SBE who have minimal risk of subsequent recurrence. If the recurrence risk after an SBE is relatively high, then perhaps clinicians should be more inclined to recommend AET following an SBE. It would be helpful to know whether the use of AET for an initial DCIS diagnosis should impact the decision regarding its use for an SBE. Future studies should address unanswered questions regarding the use of surgery, RT, and AET for the increasing cohort of woman at risk of developing SBEs.

\section{References}

1. Virnig BA, Tuttle TM, Shamliyan T, et al. Ductal carcinoma in situ of the breast: a systematic review of incidence, treatment, and outcomes. J Natl Cancer Inst 2010;102:170-178.

2. Zujewski JA, Harlan LC, Morrell DM, et al. Ductal carcinoma in situ: trends in treatment over time in the US. Breast Cancer Res Treat 2011;127:251-257.

3. Goodwin A, Parker S, Ghersi D, Wilcken N. Post-operative radiotherapy for ductal carcinoma in situ of the breast-a systematic review of the randomised trials. Breast 2009;18:143-149.

4. Staley H, McCallum I, Bruce J. Postoperative tamoxifen for ductal carcinoma in situ. Cochrane Database Syst Rev 2012;10:CD007847.

5. Gradishar WJ, Anderson BO, Aft R, et al. NCCN Clinical Practice Guidelines in Oncology for Breast Cancer. Version 1.2017. To view the

most recent version of these guidelines, visit NCCN.org. Accessed June $1,2017$.

6. Cuzick J, Sestak I, Pinder SE, et al. Effect of tamoxifen and radiotherapy in women with locally excised ductal carcinoma in situ: long-term results from the UK/ANZ DCIS trial. Lancet Oncol 2011;12:21-29.

7. Goodwin A, Parker S, Ghersi D, et al. Post-operative radiotherapy for ductal carcinoma in situ of the breast. Cochrane Database Syst Rev 2013;11:CD000563.

8. Hughes LL, Wang M, Page DL, et al. Local excision alone without irradiation for ductal carcinoma in situ of the breast: a trial of the Eastern Cooperative Oncology Group. J Clin Oncol 2009;27:5319-5324.

9. Houghton J, George WD, Cuzick J, et al. Radiotherapy and tamoxifen in women with completely excised ductal carcinoma in situ of the breast in the UK, Australia, and New Zealand: randomised controlled trial. Lancet 2003;362:95-102. 
10. Fisher B, Dignam J, Wolmark $\mathrm{N}$, et al. Tamoxifen in treatment of intraductal breast cancer: National Surgical Adjuvant Breast and Bowel Project B-24 randomised controlled trial. Lancet 1999;353:1993-2000.

11. Allred DC, Anderson SJ, Paik S, et al. Adjuvant tamoxifen reduces subsequent breast cancer in women with estrogen receptor-positive ductal carcinoma in situ: a study based on NSABP protocol B-24. J Clin Oncol 2012;30:1268-1273.

12. Yen TW, Kuerer HM, Ottesen RA, et al. Impact of randomized clinical trial results in the national comprehensive cancer network on the use of tamoxifen after breast surgery for ductal carcinoma in situ. J Clin Oncol 2007; 25:3251-3258.

13. Rakovitch E, Pignol JP, Chartier C, et al. The management of ductal carcinoma in situ of the breast: a screened population-based analysis. Breast Cancer Res Treat 2007;101:335-347.

14. Bijker N, Meijnen P, Peterse JL, et al. Breast-conserving treatment with or without radiotherapy in ductal carcinoma-in-situ: ten-year results of European Organisation for Research and Treatment of Cancer randomized phase III trial 10853 - a study by the EORTC Breast Cancer Cooperative Group and EORTC Radiotherapy Group. J Clin Oncol 2006;24:33813387.

15. Emdin SO, Granstrand B, Ringberg A, et al. SweDCIS: radiotherapy after sector resection for ductal carcinoma in situ of the breast. Results of a randomised trial in a population offered mammography screening. Acta Oncol 2006;45:536-543.

16. Habel LA, Daling JR, Newcomb PA, et al. Risk of recurrence after ductal carcinoma in situ of the breast. Cancer Epidemiol Biomarkers Prev 1998;7:689-696.

17. Arvold ND, Punglia RS, Hughes ME, et al. Pathologic characteristics of second breast cancers after breast conservation for ductal carcinoma in situ. Cancer 2012;118:6022-6030.

18. Hassett MJ, Jiang W, Habel LA, et al. Characteristics of second breast events among women treated with breast-conserving surgery for DCIS in the community. Breast Cancer Res Treat 2016;155:541-549.
19. Weeks J. Outcomes assessment in the NCCN: 1998 update. National Comprehensive Cancer Network. Oncology (Huntington) 1999;13:69-71.

20. Charlson ME, Pompei P, Ales KL, et al. A new method of classifying prognostic comorbidity in longitudinal studies: development and validation. J Chronic Dis 1987;40:373-383.

21. Katz JN, Chang LC, Sangha O, et al. Can comorbidity be measured by questionnaire rather than medical record review? Medical Care 1996;34:73-84.

22. Nichols HB, Bowles EJ, Islam J, et al. Tamoxifen initiation after ductal carcinoma in situ. Oncologist 2016;21:134-140.

23. Flanagan MR, Rendi MH, Gadi VK, et al. Adjuvant endocrine therapy in patients with ductal carcinoma in situ: a population-based retrospective analysis from 2005 to 2012 in the National Cancer Data Base. Ann Surg Oncol 2015;22:3264-3272.

24. Worni M, Akushevich I, Greenup R, et al. Trends in treatment patterns and outcomes for ductal carcinoma in situ. J Natl Cancer Inst 2015;107:djv263.

25. Habel LA, Achacoso NS, Haque R, et al. Declining recurrence among ductal carcinoma in situ patients treated with breast-conserving surgery in the community setting. Breast Cancer Res 2009;11:R85.

26. Fisher ER, Land SR, Saad RS, et al. Pathologic variables predictive of breast events in patients with ductal carcinoma in situ. Am J Clin Pathol 2007;128:86-91.

27. van de Water W, Bastiaannet E, Scholten AN, et al. Breast-conserving surgery with or without radiotherapy in older breast patients with early stage breast cancer: a systematic review and meta-analysis. Ann Surg Oncol 2014;21:786-794.

28. Yung RL, Hassett MJ, Chen $K$, et al. Initiation of adjuvant hormone therapy by Medicaid insured women with nonmetastatic breast cancer. J Natl Cancer Inst 2012;104:1102-1105.

29. Punglia RS, Burstein HJ, Weeks JC. Radiation therapy for ductal carcinoma in situ: a decision analysis. Cancer 2012;118:603-611.

30. Youngwirth LM, Boughey JC, Hwang ES. Surgery versus monitoring and endocrine therapy for low-risk DCIS: the COMET trial. Bull Am Coll Surg 2017;102:62-63.

\section{See JNCCN.org for supplemental online content.}

